\title{
Neurocinematics: The Neuroscience of Film
}

\author{
Uri Hasson, Ohad Landesman, Barbara Knappmeyer, \\ Ignacio Vallines, Nava Rubin, and David J. Heeger
}

\begin{abstract}
This article describes a new method for assessing the effect of a given film on viewers' brain activity. Brain activity was measured using functional magnetic resonance imaging (fMRI) during free viewing of films, and inter-subject correlation analysis (ISC) was used to assess similarities in the spatiotemporal responses across viewers' brains during movie watching. Our results demonstrate that some films can exert considerable control over brain activity and eye movements. However, this was not the case for all types of motion picture sequences, and the level of control over viewers' brain activity differed as a function of movie content, editing, and directing style. We propose that ISC may be useful to film studies by providing a quantitative neuroscientific assessment of the impact of different styles of filmmaking on viewers' brains, and a valuable method for the film industry to better assess its products. Finally, we suggest that this method brings together two separate and largely unrelated disciplines, cognitive neuroscience and film studies, and may open the way for a new interdisciplinary field of "neurocinematic" studies.
\end{abstract}

Keywords: fMRI, inter-subject correlation, cognitive film theory, social neuroscience, cognitive control

\section{Introduction}

Cinema takes viewers through an experience that evolves over time, grabbing their attention and triggering a sequence of perceptual, cognitive, and emotional processes. Throughout the years filmmakers have developed an arsenal of cinematic devices (e.g., montage,' continuity editing, close-up) to direct viewers' minds during movie watching. These techniques, which constitute the formal structure and aesthetics of any given cinematic text, determine how viewers respond to the film. While the idea that movies can have a tight grip on viewers' minds has been acknowledged since the early days of cinema, ${ }^{2}$ until the advent of non-invasive neuro imaging methods in the early nineties there was no way to penetrate a viewer's mind and record his or her mental states while watching a movie. 
The latest advances in functional magnetic resonance imaging (fMRI) offer an opportunity to measure brain activity during free viewing of films. fMRI utilizes a magnetic resonance imaging (MRI) scanner, like that used routinely for clinical evaluation of human anatomy, which is reprogrammed to get a time-series of 3D images of brain activity in addition to brain anatomy (Heeger and Ress 2002; Huettel, Song, and McCarthy 2004). fMRI has revolutionized neuroscience over the past decade. It has enabled a new era of research into the function and dysfunction of the human brain, complementary to more invasive techniques for measuring neural activity in animal models. In a typical fMRI experiment, a time-series of brain activity images is collected while a stimulus or cognitive task is systematically varied. If there is a large enough increase in neural activity in a certain brain region, then the image intensities in that region of the brain will increase (by as much as $\pm 5 \%$ but typically less) for a period of time following the stimulus or task that evoked the change in neural activity.

Functional magnetic resonance imaging has been used to measure brain activity primarily in the context of highly controlled experiments with extremely simple stimuli. Due to the spatiotemporal complexity of a movie sequence, conventional hypothesis-driven fMRI data analysis methods are largely unsuitable for handling the data acquired during movie watching. We therefore introduced a new method of inter-subject correlation (ISC) analysis that measures similarities in brain activity across viewers (Hasson et al. 2004). The ISC compares the response time course in each brain region (e.g., in a small region of the visual system of the brain) from one viewer to the response time courses obtained in the same brain region from other viewers (Figure 1). Because all viewers were exposed to the same film, computing ISC on a region-by-region basis identifies brain regions in which the response time courses were similar across viewers. Inter-subject correlation analysis has been utilized previously for studying the temporal scale of neural processing, the neural basis of inter-group differences, social cognition, memory, and learning (Furman et al. 2007; Golland et al. 2007; Hasson and Malach 2006; Hasson et al. 2004; Hasson, Yang et al. 2008; Hasson, Furman et al. 2008; Wilson, Molnar-Szakacs, and lacoboni 2007).

There are two important implications of the finding that a movie can evoke similar time courses of brain activity across viewers. First, some films have the potency to "control" viewers' neural responses. By "control" we simply mean that the sequence of neural states evoked by the movie is reliable and predictable, without placing any aesthetic or ethical judgment as to whether the means to such control are desirable. Second, under the assumption that mental states are tightly related to brain states (a hypothesis that is widely believed to be true by most neuroscientists and many philosophers), controlling viewers' brain states, for our purposes, is the same as controlling 
their mental states including their percepts, emotions, thoughts, attitudes, etc. (Crick 1994; Damasio 2000; Ledoux 1998).

This article proposes to utilize ISC for measuring the effectiveness of popular media on viewers' brains. The idea is relatively simple and straightforward. In cinema, some films (or films' segments) lead most viewers through a similar sequence of perceptual, emotional, and cognitive states. Such a tight grip on viewers' minds will be reflected in the similarity of the brain activity (high ISC) across most viewers. By contrast, other films exert (either intentionally or unintentionally) less control over viewers' responses during movie watching (e.g., less control of viewers' emotions or thoughts). In such cases we expect that there will be less control over viewer's brain activity; that is, more variability across viewers (low ISC). For example, high ISC in visual or auditory areas in response to a given movie sequence implies a high effectiveness of the visual image or soundtrack on viewers' visual or auditory percepts, respectively. Likewise, high ISC in brain areas related to emotion processes or cognitive processes assesses the effectiveness of a movie in controlling viewers' emotions and thoughts, respectively.

\section{Measuring Inter-Subject Correlation During Film Viewing}

In our first study of brain activity during movie watching, we asked five volunteers to view the opening 30 minutes of The Good, the Bad and the Ugly, a well-known film by Sergio Leone (1966), while their brains were scanned with fMRI (Hasson et al. 2004). The volunteers lay on their backs in the MRI scanner. Digitized video and sound were supplied by a computer system. The video was presented with an LCD projector on a screen behind the volunteers' heads, and was viewed via a mirror mounted over their eyes. Sound was delivered via high fidelity MRI-compatible earphones (Figure $1 \mathrm{~A}$ ). The volunteers were simply asked to watch the movie. They were free to choose what to look at, although they were asked to hold their heads still, and they had the option of ending the movie and getting out of the scanner at any time during the experiment. The fMRI data were processed by computationally registering each viewer's brain into what is known as the Talairach coordinate system, so that corresponding regions of each brain were roughly aligned with one another, spatially smoothing the data to overcome any residual misregistration between brains, and then correlating the response time courses in a given brain region across viewers (Figure $1 \mathrm{~B}$ ).

Despite the seemingly uncontrolled (free viewing) task and complex nature of the movie stimulus, brain activity was similar across viewers' brains. Specifically, about 45 percent of the neocortex (the folded surface of the brain, henceforth cortex) showed high (and statistically significant, $p<0.001$ ) intersubject correlation during movie watching (Figure 2A). The correlation covered many different brain regions, including visual areas in the occipital and tem- 
Figure 1. Inter-Subject Correlation Analysis.

Figure 1 A. Volunteers watched movies and TV episodes while their brain activity was recorded with fMRI.

Figure $1 B$. The ISC analysis measures similarity in brain activity across viewers by comparing the response time course in each brain region from one viewer with the response time courses obtained in the same brain region from other viewers during movie watching.
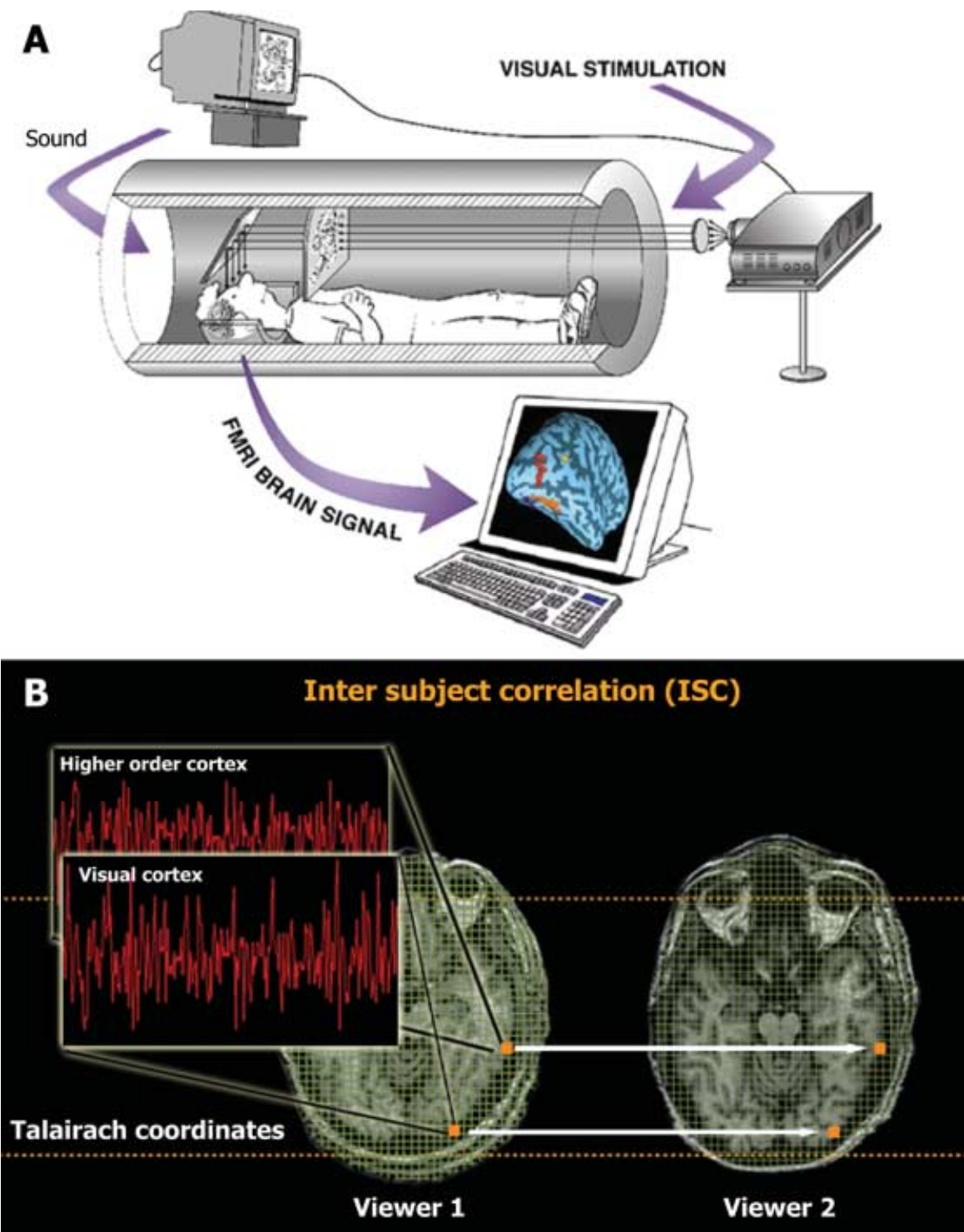

poral lobes of the brain, auditory areas in Heschl's gyrus, regions near the lateral sulcus known to be critical for language (also known as Wernicke's area), brain regions that have been implicated in emotion, and multi-sensory areas in the temporal and parietal lobes. The strength of the ISC can be appreciated by inspecting the response time courses in each of these brain regions. Figure $2 \mathrm{~B}$, for example, plots the response time courses sampled from the fusiform face area (FFA) - a region of the brain believed to be critical for face recognition (Kanwisher, McDermott, and Chun 1997; Kanwisher and Moscovitch 2000)across all five viewers. The activity in this brain area increased and decreased 


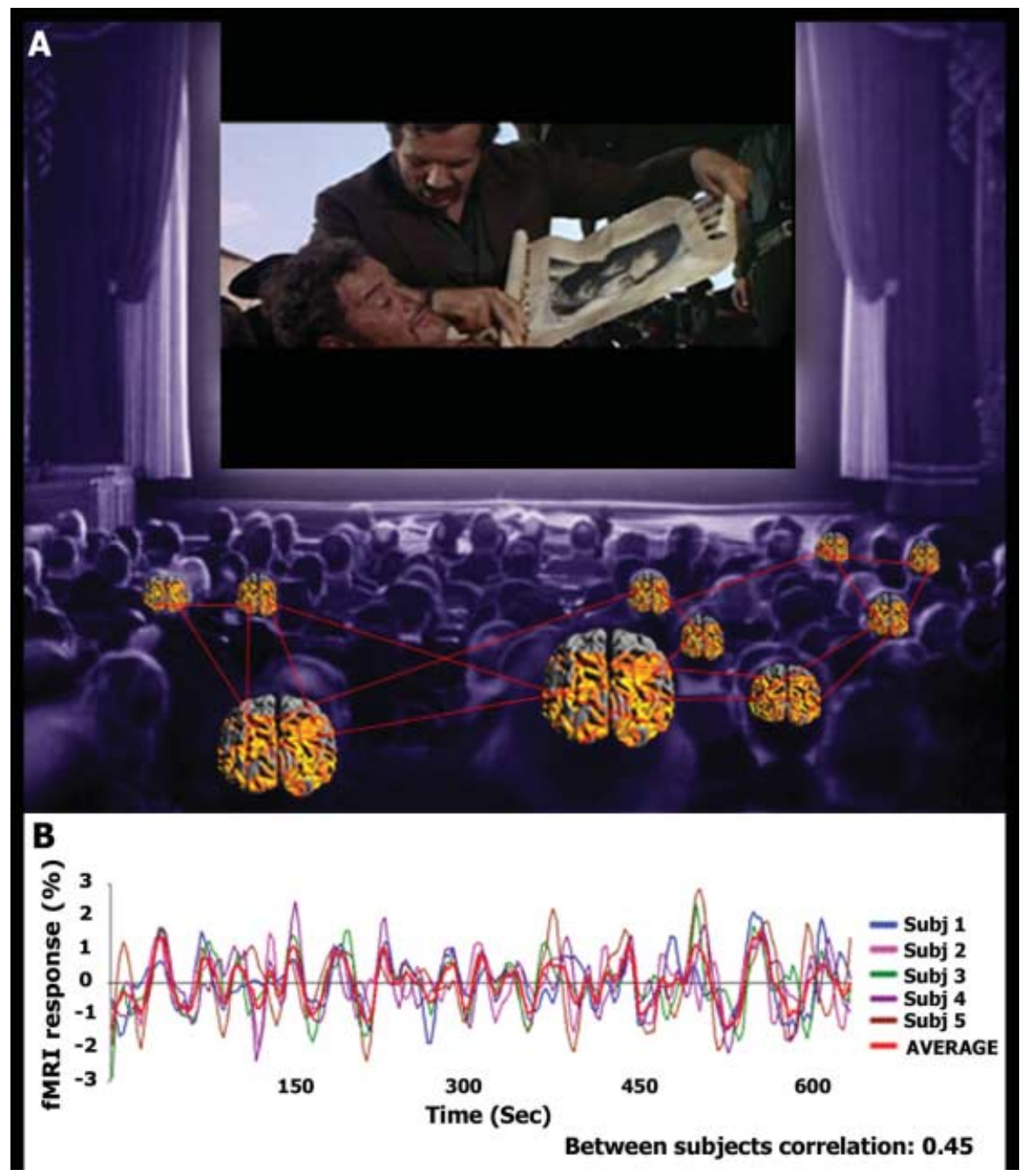

Figure 2. Inter-subject correlation in brain activity to an example commercial film.

Figure 2A. Sergio Leone's The Good, the Bad and the Ugly (1966) movie evoked similar responses across all viewers in about 45 percent of the cortex during movie watching.

Figure 2B. The similarity in brain activity can be appreciated by inspecting the fMRI response time courses sampled from a representative brain area (fusiform face area) for all five viewers.

following a similar time course in all viewers during movie watching. In other words, the movie exerted considerable control over the responses of this brain area, evoking a similar time course of activity. Graphs like Figure 2B could be shown for many brain regions (colored in orange in Figure $2 \mathrm{~A}$ ), each of which exhibited a response time course that was similar across viewers.

The movie exerted considerable control over the responses of

In addition to the high ISC in brain activity, we also this brain area, evoking a similar found that the same film exerted considerable control over viewers' behavior as measured by tracking their eye movetime course of activity. ments (Figures $3 \mathrm{~A}$ and $3 \mathrm{~B}$ ). The viewers were free to look anywhere as there were no instructions other than to lie still in the MRI scanner and watch the movie, but for many scenes all viewers fixated on the same location at the 
Figure 3. Inter-subject correlation in eye position.

Figure 3 A. Horizontal eye position from four viewers watching The Good, the Bad and the Ugly (recorded concurrently with fMRI).

Figure $3 B$. Vertical eye position from the same viewers and movie. Note the high degree of agreement in the eye movement traces across viewers.

\section{Eye movements}

\section{The Good the bad and the Ugly}

\section{A Horizontal eye position}
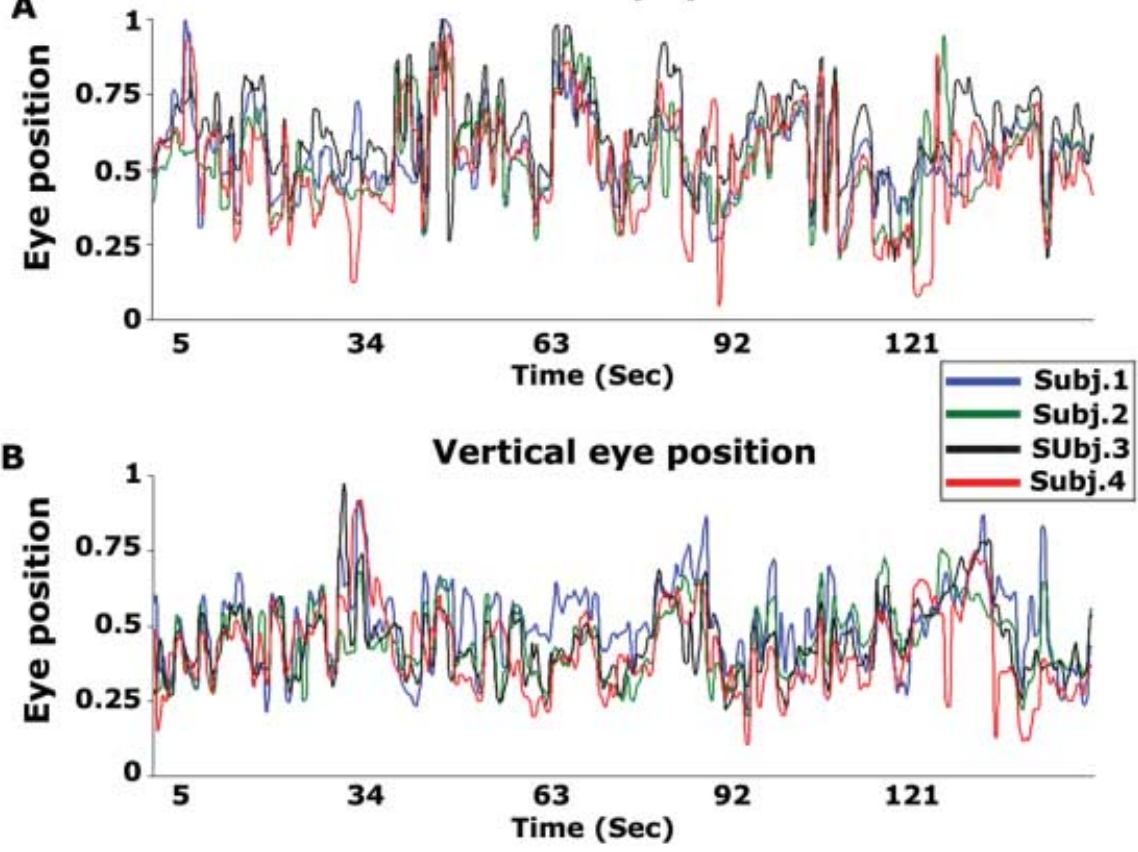

C Structured movie

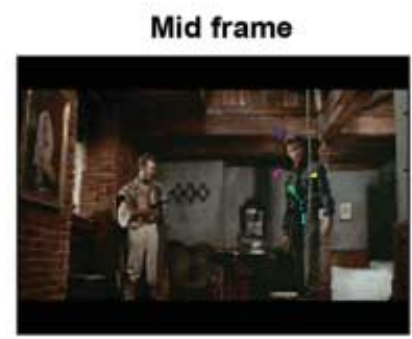

Average Gaze map

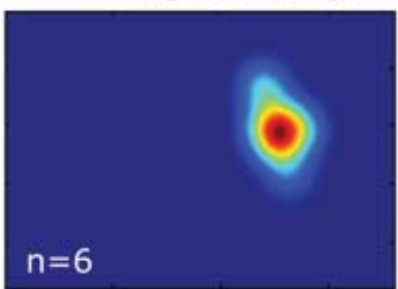

Figure 3C. Typical example of an average gaze map across six viewers of The Good, the Bad and the Ugly. Top, frame from a 0.5 second duration clip. Bottom, color represents the total time spent fixating on each location in the frame summed across all viewers. The single red spot means that viewers fixated primarily on the same location during this clip.

\section{Unstructured movie}

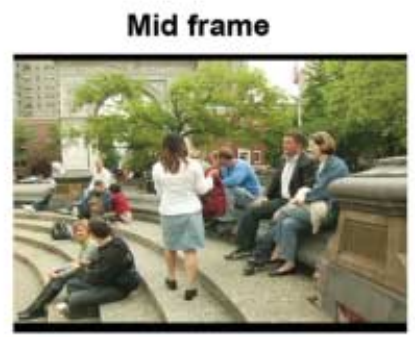

\section{Average Gaze map}

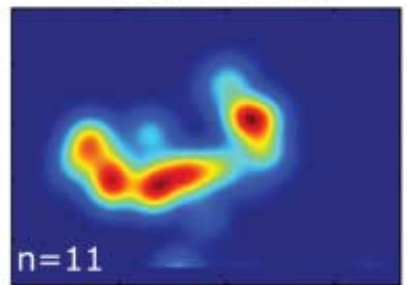

Figure 3D. Typical example of an average gaze map for the unstructured, one-shot, segment of reality video filmed in Washington Square Park. Top, frame from a 0.5 second duration clip. Bottom, the multiple red spots mean that viewers looked at different elements during this clip. 
same time (Figure $3 \mathrm{C}$ ). The relationships between the ISC in brain activity and eye positions are discussed below.

\section{Specificity and Selectivity of Inter-Subject Correlation}

Does the high and widespread inter-subject correlation attest to the engaging power of this particular film's structure, or is it an accidental epiphenomenon that can tell us nothing about the characteristics of this movie? To isolate the intervening dimensions that drive the ISC in each brain region we systematically manipulated different aspects of the movie sequence.

First, we established that the similarity in response time courses across viewers was induced by the content of the movie. Measuring the ISC between brains while viewers were in complete darkness revealed no evidence for such correlation across viewers' brains (Hasson et al. 2004). Similarly, no evidence for ISC was found for viewers who watched different segments of the same movie. These data suggest that the observed similarity in brain activity was time locked to, and hence, induced by the particular sequence of events in the movie. In other words, the same course of events can cause us (and our brains), in some cases, to respond in a similar way (Figure 2). However, in the absence of external stimulation (as in darkness), or when exposed to different sequences of events (different segments of the same movie), our brains respond differently.

Second, we established that the ISC depended on the characteristics of a particular movie sequence, and that high levels of ISC were not obtained for all kinds of motion pictures. Movies depict complex events. On the one hand, merely exposing viewers to the same sequence of events might, to some extent, induce similar responses in their brains. If this were the case then we would expect high ISC for any type of a movie sequence, independent of the movie's content and directing style. On the other hand, the richness and complexity of real life events might evoke very different responses across viewers, because each individual may perceive and process the same situation in a different manner. If this were the case then we would expect the level of ISC to vary as a function of the level of control a movie has on viewers' mental states, and to be less apparent in real life open-ended situations. To distinguish between these two possibilities, we presented viewers with a 10-minute, unedited, one-shot video clip of a Sunday morning concert in Washington Square Park in New York City. The one-shot was taken from a fixed single view point, letting people come in and out of the frame without any intervention on our part. Thus, in this experiment, we compared the ISC for an unstructured real life event (filmed without employing any cinematic devices such as pans, cuts, and close-ups), with the ISC for a tightly edited and influential commercial film.

The unstructured, one-shot video evoked far less ISC across viewers than Sergio Leone's The Good, The Bad and the Ugly film (Figure 4, blue indicates 


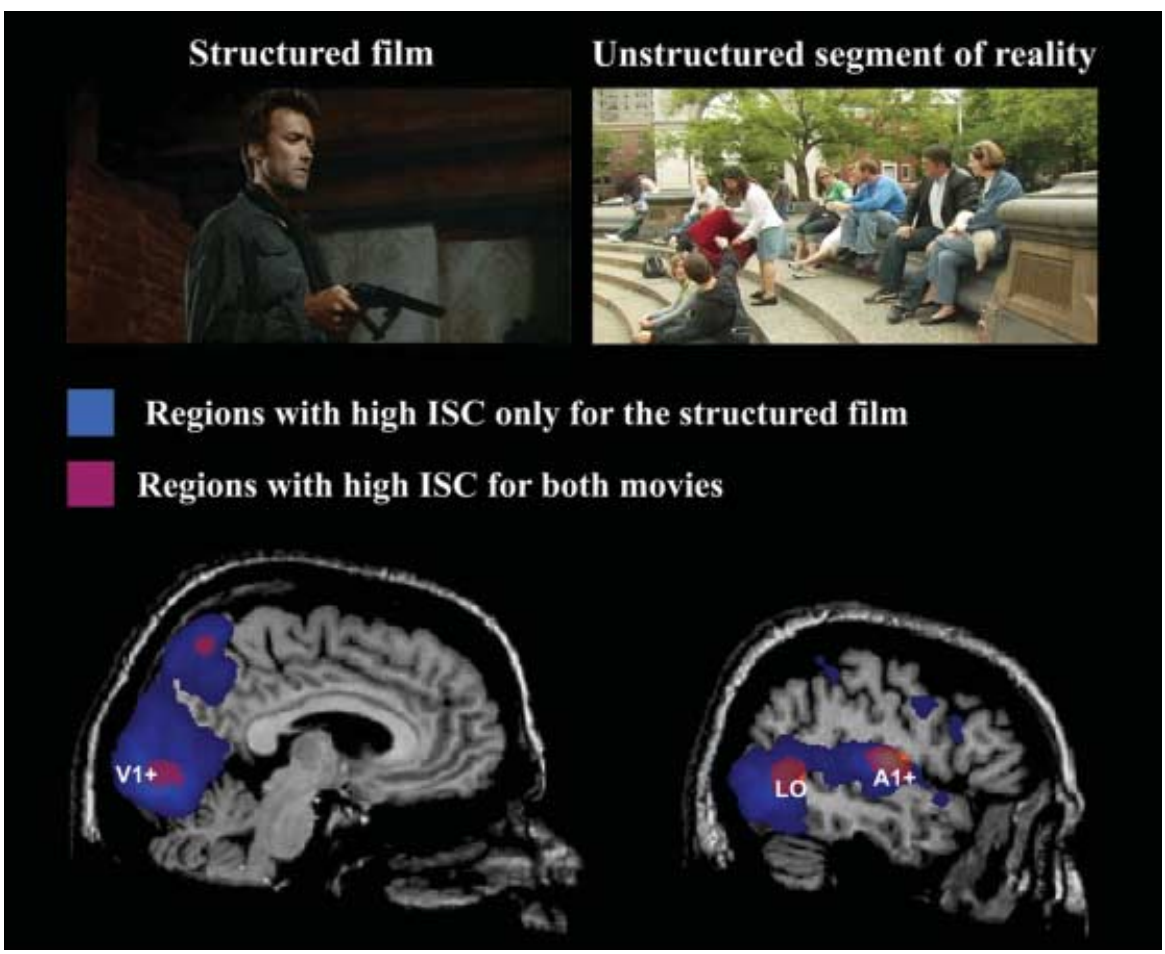

Figure 4. Inter-subject correlation for structured and unstructured films.

The ISC for The Good, the Bad and the Ugly and for the unstructured, one-shot, segment of reality filmed in Washington Square Park. Blue marks regions with high ISC for only the The Good, the Bad and the Ugly. Purple marks regions with high ISC for both movies. The unstructured one-shot evoked far less ISC than The Good, the Bad and the Ugly. V1+ indicates the approximate location of primary visual cortex, the main target for sensory input from the eyes. A1+ indicates the approximate location of primary auditory cortex, the main target for input from the ears. LO indicates a region of lateral occipital cortex that is known to be involved in visual cognition and object recognition.

brain regions that exhibited high ISC only for the Sergio Leone film and purple indicates high ISC for both movies). The ISC was still high in a few visual (labeled $V_{1}+$ in the Figure) and auditory (labeled $A_{1}+$ ) areas and in an area of the lateral occipital lobe (labeled LO) that is known to be involved in object recognition. But the unstructured movie evoked much less ISC than The Good, the Bad and the Ugly, particularly in regions of the brain beyond those that are known to be involved in basic sensory processing of visual and auditory input. These findings suggest that a mere mechanical reproduction of reality, with no directorial intention or intervention, is not sufficient by itself for controlling viewer's brain activity. In this experiment we presented viewers with a clip of an arbitrary sequence of events in the park. It is feasible that some other representations of real life events (e.g., an engaging lecture or a good basketball game) will have greater grip on viewers' responses, inducing higher correlation in their brain responses. However, it is also feasible that most arbi- 
trary sequences of events or shots (similar to most arbitrary combinations of words in Jorge Luis Borges famous story "The Library of Babel") will have little control over viewers' brain responses (see, for example, the scrambling experiment below). Our data suggest that achieving a tight control over viewers' brains during a movie requires, in most cases, intentional construction of the film's sequence through aesthetic means.

Third, we established that the ISC dissociated brain activity to the film's images from brain activity to the film's soundtrack. Moreover, the ISC identified multi-modal brain regions, which perform cognitive processing that is independent of the mode of presentation (visual or audio). Films are composed of a sequence of audio-visual stimuli. To dissociate the neuronal responses to the film's images from the neuronal responses to the soundtrack, we compared the ISC for a well-structured and visually guided movie from which we removed the soundtrack (Charlie Chaplin's classic film City Lights, 1931) with the ISC for a well structured segment of an audio-book soundtrack (Lewis Carroll's classic book Alice in Wonderland). The results revealed a strong specificity, in which visual cortex was highly correlated across viewers during the movie with its soundtrack removed (Figure 5, red), but not during story listening, and vice versa for auditory cortex (Figure 5, yellow). This comparison also revealed overlapping regions of high ISC (Figure 5, orange) in the superior temporal sulcus (STS), temporal-parietal junction (TPJ), and part of the left intraparietal sulcus (IPS). These multisensory regions of the brain may be involved in more abstract forms of processing (e.g., processing sequences of events, human interactions, narrative) shared by the visual film and audio book.

Figure 5. Distinct inter-subject correlations for the visual image and audio soundtrack. The ISC for a movie with its soundtrack removed (City Lights) and for an audio-book soundtrack (Alice in Wonderland). Note the strong specificity in which visual areas were highly correlated across viewers during movie watching (red), but not during story listening, and vice versa for auditory cortex (yellow). Anatomical abbreviations in white: STS- superior temporal sulcus; TPJ-temporalparietal junction; IPS - intraparietal sulcus. Functional abbreviations in black: V1+- approximate location of primary visual cortex; A1+-approximate location of primary auditory cortex.

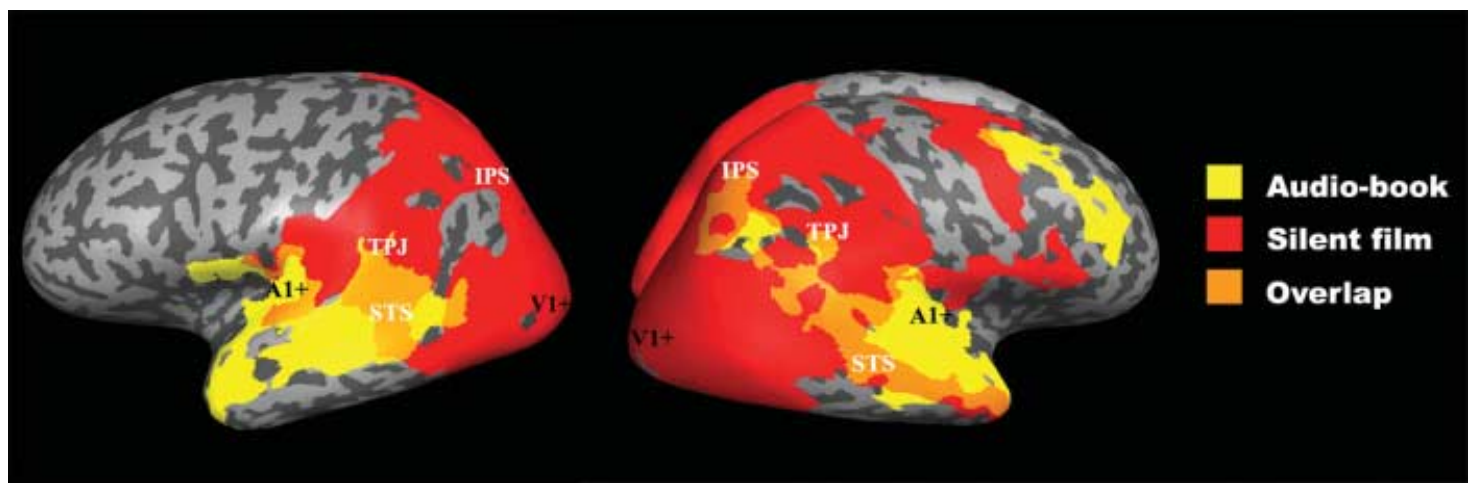




\section{From Single Shots to Juxtaposition of Shots to Coherent Movie Sequences}

A movie is not just a mere collection of isolated elements. For a movie to be effective, it is not enough for it to include a succession of individual shots (the basic building blocks of the movie sequence) and sound elements. Rather, a meticulous editing of the individual shots and sounds is needed to combine all these pieces into a coherent whole.

To assess the effect of editing (juxtaposition of adjacent individual shots) on viewers, we varied the temporal structure of a movie sequence (Hasson, Yang et al. 2008). This was done by marking the film's single shots, as defined by the movie's editor, and then randomly shuffling the order of these individual shots at three temporal scales: short, intermediate, and long. We used Charlie Chaplin's films (The Adventurer, 1917 and City Lights, 1931) with any soundtracks removed, to avoid the added complexities of editing the soundtrack. The randomized editing procedure created, from the same shots, four different movie sequences with varying degrees of temporal coherency. A) The original unscrambled movie sequence possessed the most coherent temporal structure. B) The long time-scale movie sequence preserved temporal coherency over approximately 36 -second segments (each composed of around 8-10 shots) but lost the temporal coherency over longer periods of time. C) The intermediate time-scale movie sequence preserved temporal coherency only within approximately 12 -second segments (each composed of around 3-4 shots). D) The short time-scale movie sequence was composed of a randomly shuffled sequence of single shots, each approximately 4 seconds in duration, that seemed unrelated to each other.

The original unscrambled movie as well as each of the scrambled movies were presented twice. This design allowed us to measure the correlation of the responses across repeated presentations of the same movie. For details of the analysis and for a direct comparison with the ISC analysis described above, see Hasson, Yang et al. (2008). In brain areas where responses are driven primarily by the instantaneous sensory input, the responses should be similar across repeated presentations of each movie, regardless of its temporal coherency. In contrast, in brain regions where responses depend on the temporal coherency of the edited sequence, the correlation across repeated presentations should depend on the time-scale of scrambling.

The results revealed differences across brain regions as a function of temporal coherency of the edited sequence (Figure 6). The correlation across repeated presentations in some visual areas (labeled $V_{1}+$ and $M T+$ in Figure 6A; blue in Figure 6B) was high for each of the three scrambled films and was similar to the level of ISC evoked by the original unscrambled film (i.e., all four colored bars in Figure $6 \mathrm{~A}$ are high for $\mathrm{V}_{1}+$ and $\left.\mathrm{MT}+\right)$. The correlation across repeated presentations in these visual areas was not affected by disturbing the temporal structure of the movie, demonstrating that the brain activity in 

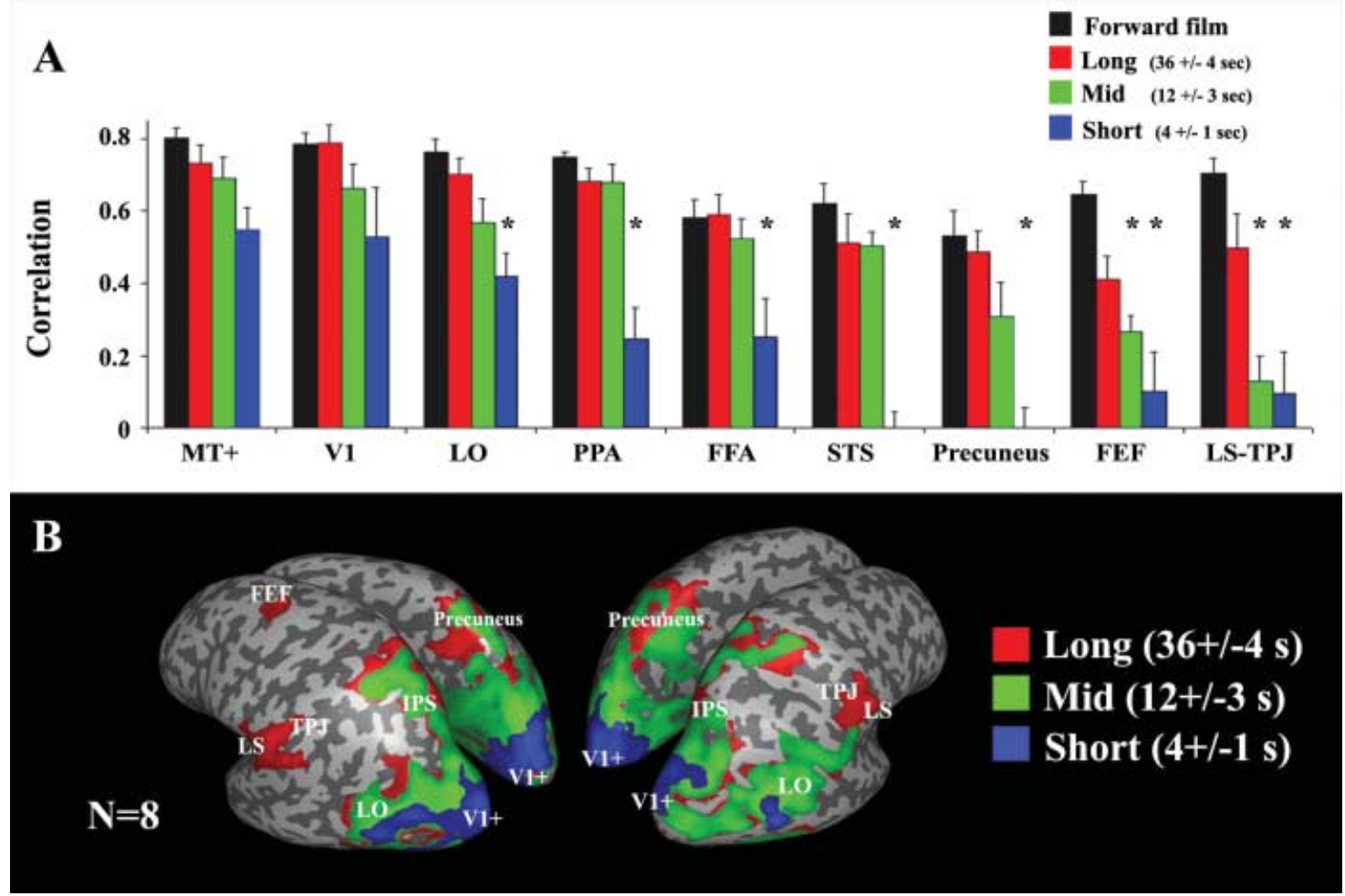

Figure 6. Response similarity as a function of temporal coherency.

Figure 6A. The correlation of the responses across repeated presentations of the same movie in each of several brain regions (V1+, MT+, LO, PPA, STS, precuneus, LS-TPJ, and FEF) for four different levels of temporal coherency. This was done by marking the film's single shots, as defined by the movie's editor, and then randomly shuffling the order of these individual shots at three temporal scales. Black bars - the response correlations for the unscrambled original movie that had the most coherent temporal structure. Red - the response correlations for the longest time scale of

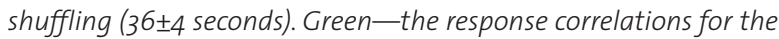
intermediate time scale of shuffling (12 \pm 3 seconds). Blue-the response correlations for the shortest time scale of shuffling ( $4 \pm 1$ seconds). Asterisks indicate that the response correlations were (statistically significantly) smaller than that evoked by the unscrambled original version.
Figure 6B. Brain regions varying in their dependence on temporal coherency. Bluebrain regions in which the response correlations were high for all shuffled movies (at long, intermediate and short time scales). Green-regions in which the response correlations were high only for the long and intermediate time scales, but not when the shots were shuffled at a short time scale (e.g., LO, PPA, FFA, STS above). Red-regions in which the response correlations were high only for the longest time scales (e.g., LS, TPJ, and FEF).

these areas was driven mainly by the momentary visuospatial content of the individual shots, regardless of the overall temporal coherency (or temporal relationship between shots). An intermediate temporal coherency (approximately 12 seconds, which consists of the juxtaposition of around 3-4 shots in our experiments) was needed to drive high correlation across repeated presentations in other brain regions such as the lateral occipital (LO) cortex, parahippocampal place area (PPA), fusiform face area (FFA), superior-temporal 
sulcus (STS), and precuneus. This is indicated by green in Figure $6 \mathrm{~B}$ and by brain areas with short blue bars but tall green, red, and black bars in Figure 6A. Although the exact functions of these brain regions are still under investigation, such intermediate temporal coherency may be necessary for visual and cognitive processing functions that require integration of information across events. An example might be the processing of different kinds of montage relationships between adjacent shots (Eisenstein 1925; see also Mobbs et al. 2006). Finally, processing over a long temporal scale (ranging from around 30 seconds to the entire movie sequence in our experiments) was needed to achieve high correlation across repeated presentations in more anterior regions of the brain that are believed to perform more complex, cognitive functions. This is indicated by red in Figure $6 \mathrm{~B}$ and by brain areas with tall red and black bars but short blue and green bars in Figure 6A: the lateral sulcus (LS), temporal parietal junction (TPJ), and frontal eyefield (FEF). Such long temporal coherency may be necessary for cognitive functions related to the processing of the movie as a whole; for example, for inferring the characters motives, intentions, and beliefs, and for processing the plot and predicting outcomes. ${ }^{3}$

These results show that re-editing the exact same list of shots can have a dramatic effect on the responses in brain areas subserving cognitive functions (which accumulate information across shots and process the movie as a whole), but with little effect on the responses in sensory brain areas (which process the instantaneous information within single shots).

\section{Attention and Eye Movements}

To what extent do the inter-subject correlations in brain activity depend on the success of the filmmaker in controlling what viewers are looking at and attending to (Figure 3 )? The outside world is complex and the computational resources of our brains are limited. Thus, the brain relies on attentional mechanisms to select what appears to be the most relevant information for further processing. Filmmakers utilize a number of cinematic devices (lighting, composition and framing of a shot, movement or lack of movement, etc.) to control the salience of certain locations in each shot, hence controlling viewers' attention and eye movements. The idea of framing reality in cinema presupposes an act of exclusion and inclusion that is intended to channel the viewer's gaze and attention in a predetermined and controlled manner. ${ }^{4}$

Viewers tended to fixate on similar objects within each shot at about the same time while watching the Sergio Leone film (Figure $3 \mathrm{~A}-\mathrm{C}$ ). For example, in the scene depicted in Figure $3 \mathrm{C}$, the gaze of all viewers was guided to the actions of the protagonist on the right side of the frame despite the complex spatial layout of the scene. However, this was not the case for the one-shot Washington Square video, in which viewers' attention was not guided by the filmmaker. An analysis of the eye movements during the unstructured one- 
shot revealed that viewers chose to attend to different objects at each moment (see Figure 3D for a representative example). If a filmmaker fails to direct viewers' gaze, then each viewer will attend to and process different information at each moment in time, which will subsequently increase the variability in brain responses across viewers. This may correspond to increased variability across viewers in the interpretation of the scene that may, in turn, lead to increased variability in the interpretation of subsequent scenes. Thus, an assessment of the scene-by-scene control of a given movie sequence upon viewers' eye movements may be of importance to filmmakers.

However, recording eye movements during movie watching, while very illuminating, is not sufficient by itself to determine the amount of control a movie has on viewers' emotional and cognitive responses. To demonstrate this, we measured concurrently eye movements and brain activity to movies (with no sound track) played forward and backward in time (Hasson, Yang et al. 2008). The eye movements were highly correlated across viewers and very similar across repeated presentations of the same movie for both the forward and backward movies (see figure 4 in Hasson, Yang et al. 2008). The brain activity in visual cortex was, likewise, highly correlated for both the forward and backward films. But the correlations in brain activity in some other cortical areas (precuneus, LS, TPJ, and FEF) were much smaller during backward than during forward presentations (see

Recording eye movements during movie watching, while very illuminating, is not sufficient by itself to determine the amount of control a movie has on viewers' emotional and cognitive responses. figure 2 in Hasson, Yang et al. 2008). The similarity of the eye movements for both forward and backward films suggests a comparable level of engagement, mitigating potential concerns that the lower correlations in brain activity during the backward movies occurred because viewers paid less attention to them. Playing the movies backward had a great impact on their intelligibility, however, as measured by a questionnaire administered to viewers after the screening (see supplementary figure 4 in Hasson, Yang et al. 2008). Not only were viewers incorrect in summarizing the plot and the intentions of the characters, but they were highly variable in their responses to the questionnaire. The high variability of activity in some brain areas to the backward films is consistent with the high variability in viewers' comprehension of the backward movies. This indicates that although necessary, it is not sufficient for all viewers to attend to the same object or event at each moment. Similar eye movements do not guarantee similar brain responses. Similar eye movements only indicate that some aspects of visual processing are correlated across individuals. Thus, measuring the correlation in brain activity (either across viewers or across repeated presentations to the same viewer) can provide complementary information for assessing the cognitive and emotional effectiveness of a movie, not provided by analysis of the eye movements. 


\section{Collective Engagement}

The ability to measure the effect of films on viewers' brains with high spatial and temporal precisions can provide a new analytical paradigm for assessing and analyzing different aspects of films, film genres, and cinematic styles.

As an initial step to test the potential of the ISC method for assessing different types of movies, we compared the ISC obtained for Sergio Leone's The Good, the Bad and the Ugly (1966) with the ISC obtained for two TV episodes, an episode of Alfred Hitchcock Presents (Bang! You're Dead, 1961, directed by Alfred Hitchcock), and an episode of Larry David's Curb Your Enthusiasm (2000). As a point of reference we also compared the results with those obtained from the one-shot Washington Square Park video. The fMRI measurements for all four movies were acquired with the same equipment and procedures.

The ability to measure the effect of films on viewers' brains with high spatial and temporal precisions can provide a new analytical paradigm for assessing and analyzing different aspects of films, film genres, and cinematic styles.
This is important because the quality of fMRI measurements (the amount of noise and variability that has nothing to do with brain activity) depends on how the data are acquired (e.g., the magnetic field strength of the MRI scanner), and lower quality (noisier) measurements would yield ostensibly lower ISC. Although the initial publication of the ISC for the Sergio Leone film (Hasson et al. 2004) was based on measurements from a different type of

MRI scanner, we repeated the experiment for the results shown in this article (Figures 2-4, 7, and 8 ) to have a fair comparison between this and the other films. In addition, to have a fair comparison, we extracted 10 minutes of fMRI measurements from the full duration of responses to each of the four movies, because the movies differed in length and the statistics of the correlation values may depend on the duration.

The extent of ISC differed for the four movies (Figure 7A). The percentage of cortex exhibiting high ISC provided a measure of the overall effectiveness, or collective engagement power, of each movie to induce similar responses across viewers (Figure 7B). The Hitchcock episode (Figure 7, green) evoked similar responses across all viewers in over 65 percent of the cortex, indicating a high level of control of this particular episode on viewers' minds. The high ISC was also extensive (45\%) for the Good, the Bad and the Ugly (Figure 7, blue), but much less so (18\%) for Curb Your Enthusiasm (Figure 7, red). Finally, as noted above, the unstructured segment of reality (Figure 7, orange; see also Figure 4) induced high ISC only in a small fraction of the cortex (less than $5 \%$ ). ${ }^{5}$

From the point of view of film studies this selection of four movies might seem arbitrary and fractional. The data were gathered from several independent cognitive neuroscience experiments, and the justification for selecting each movie for each of those experiments lies elsewhere (Furman et al. 2007; Golland et al. 2007; Hasson, Yang et al. 2008; Hasson, Furman et al. 2008; Hasson 


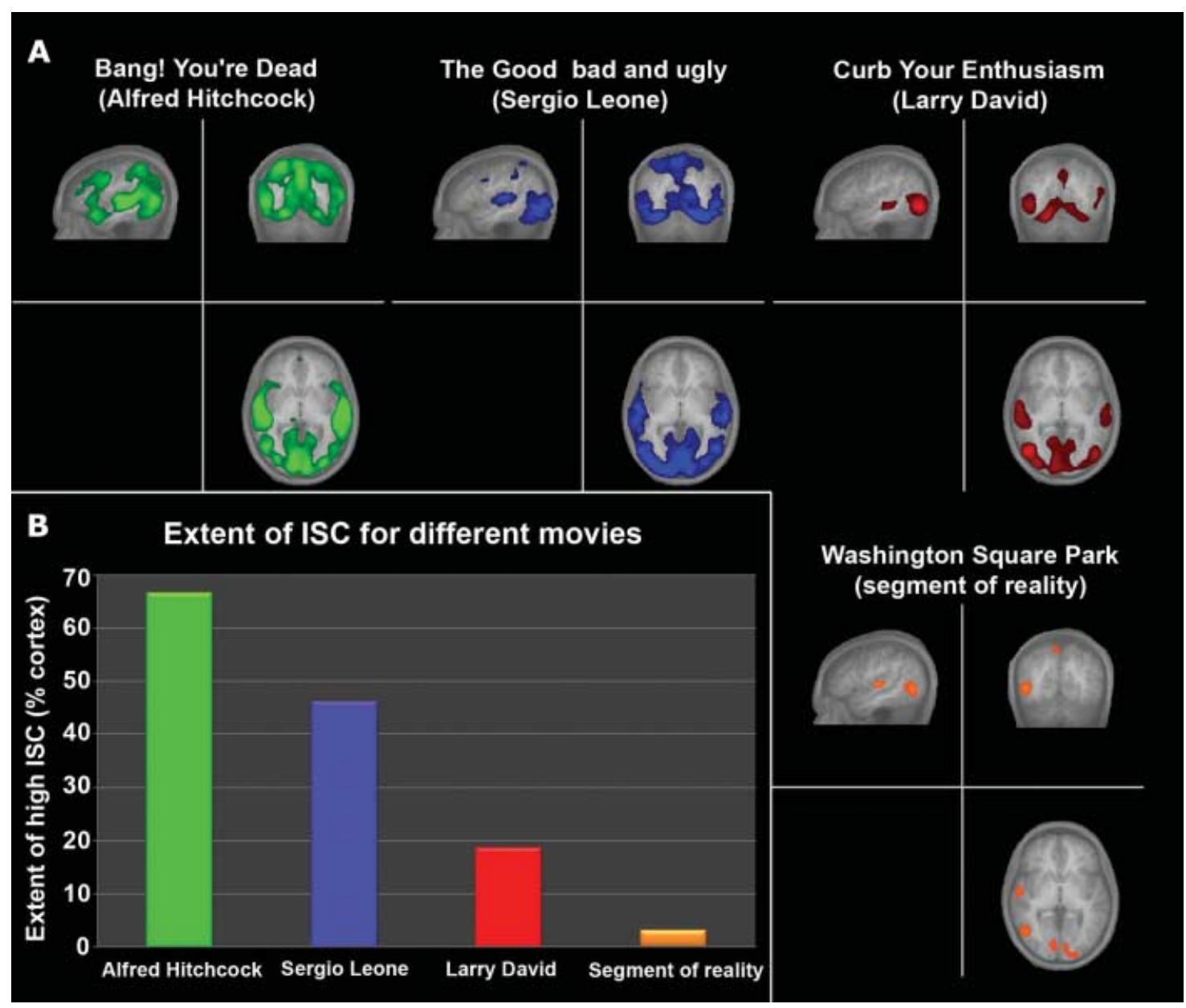

Figure 7. Inter-subject correlation for different films

Figure 7A. The ISC for four different films: Alfred Hitchcock Presents: Bang,! You're Dead (green), Sergio Leone's The Good, the Bad and the Ugly (blue), Larry David's Curb Your Enthusiasm (red), and the unedited, one-shot segment-of-reality video filmed in Washington Square Park (orange). The three images in each panel depict the ISC in typical slices through the brain at each of the three cardinal orientations.
Figure $7 B$. The extent of ISC evoked by each movie segment as measured by the percentage of cortex that exhibited high ISC.

and Malach 2006; Hasson et al. 2004). However, we do believe that the four movies differ in terms of their level of aesthetic control, and that even these initial results convey some important implications for film theory and filmmakers (as discussed next). Furthermore, one of the purposes of this article is to introduce a neuroscience-based paradigm to scholars in the field of cinema studies in an attempt to initiate a more thorough scholarly exploration of the relationship between film and cognitive neuroscience. 
The fact that Hitchcock was able to orchestrate the responses of so many different brain regions (Figure 7, green), turning them on and off at the same time across all viewers, may provide neuroscientific evidence for his notoriously famous ability to master and manipulate viewers' minds. Hitchcock often liked to tell interviewers that for him "creation is based on an exact science of audience reactions" (Douchet 1985).

Different filmmakers strive to achieve different levels of control over their viewers' reactions (see notes 1 and 2). Our findings provide empirical evidence to support the long-lasting distinction in film theory between films that remain faithful as much as possible to reality and those that seek to control or distort it. Writing about post-World War II sound cinema, film theorist and critic André Bazin rejected the traditional distinction often made between sound and silent films in favor of an innovative critical differentiation between "those directors who put their faith in the image and those who put their faith in reality" (1967: 24). Bazin distrusted the highly controlled dynamic juxtaposition of images in montage editing on ideological and ethical grounds: the more controlled the aesthetic is, the further these films manipulate the viewer with an unequivocal message (e.g., Russian montage, German Expressionism). On the other hand, films that use uninterrupted long takes, deep focus, multi-space composition, or other realistic film conventions, introduce a democratic ambiguity to the image, and invite viewers to draw their own individual conclusions. Over the years, it has become fashionable in film studies to differentiate between "Bazinian" filmmakers (e.g., directors of the European Art Cinema and Italian neo-Realism), who strive to maintain an ambiguity in the image and allow for several possible interpretations, and those filmmakers (e.g., directors of the Classical Hollywood Style), who hope to gain maximal control over viewers' mental responses by using continuity conventions and any suitable cinematic device to achieve such a goal. ${ }^{6}$ Similarly, there are documentary filmmakers who try to convey an impression of objectivity and partiality in their nonfiction representations (e.g., the American Direct Cinema filmmakers), while others borrow tools of fiction storytelling to dramatize and convey their message to as many viewers as possible (e.g., Michael Moore's films). Thus, while the ISC cannot provide an aesthetic judgment as to the right cinematic style to be taken, it may serve as an objective scientific measurement for assessing the effect of distinctive styles of filmmaking upon the brain, and therefore substantiate theoretical claims made in relation to them.

An assessment of both extremes, where films have minimal or maximal control over viewers' minds, can be illuminating (Figure 8A). At one extreme, a film that is completely and intentionally unstructured by the filmmakers is at risk of being pointless and unengaging for most viewers. Surely, the deliberate decisions taken by a filmmaker to rearrange reality in a certain way dissociate 


\section{A. Collective engagement}

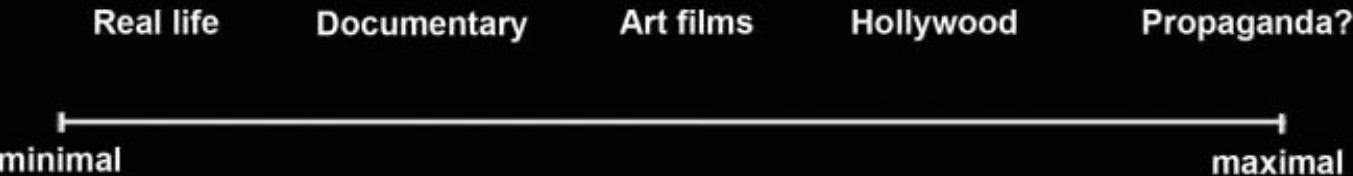

\section{The extent of ISC across the entire brain}

maximal

\section{B. Effectiveness}

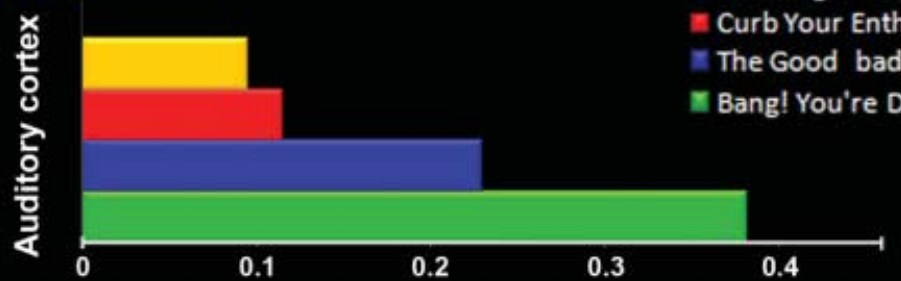

Washington Square park

n Curb Your Enthusiasm

The Good bad and ugly

Bang! You're Dead

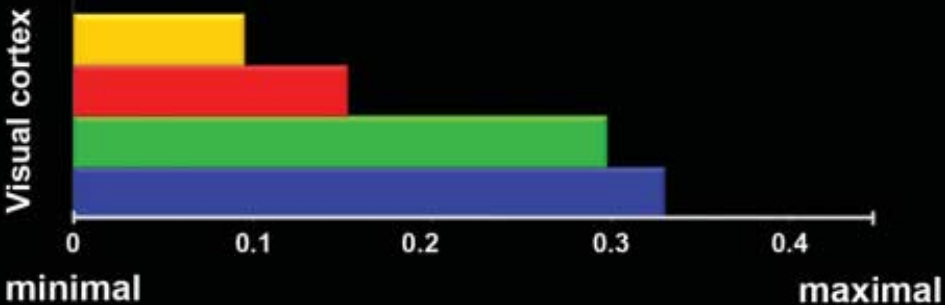

The level of ISC

Figure 8. Inter-subject correlation as a measure of the collective engagement and effectiveness of different films.

Figure 8A. The ISC can serve as an objective analytical method for assessing the level of control each film or film genre has on viewers' minds, ranging from minimal control (e.g., in unstructured segment of reality) to maximal control (e.g., in Hollywood films).
Figure 8B. Additional information about the effectiveness of different aspects of the film can be obtained by measuring the ISC separately for each of several brain regions. The ISC is plotted separately for visual cortex and auditory cortex, for each of four movies.

the art of filmmaking from being merely an act of mechanical replication of reality. ${ }^{7}$ Furthermore, we may speculate that part of the mesmerizing power of movies stems from their ability to take control of viewers' minds, and that viewers often seek and enjoy such control because it allows them to become deeply absorbed (and mentally engaged) in the movie. On the contrary, maximal control over viewers' minds might simplify and trivialize the art work; this can be seen in some popular films where the fear of losing the grip over the audience creates oversimplified or overstated films, which simply 'explain too 
much.' Finally, taken to an extreme, the possibility to achieve a tight grip on the viewers' minds can be used for creating an unethical form of propaganda or brainwash. ${ }^{8}$

\section{Qualifications, Refinements, and Extensions}

It is important to distinguish between the level of processing devoted to analyzing the incoming stimuli and the effect it has on viewers. The finding that some films have low ISC does not necessarily imply that the viewers were not attentive to nor engaged/absorbed with the events in those films. ISC measures only the ability of the filmmaker to evoke similar responses across all viewers. Similar brain activity across viewers (high ISC) can be taken as an indication that all viewers process and perceive the movie in a similar manner. Variability in the brain activity across viewers (low ISC) can be due to either a less engaged processing of the incoming information (e.g., as in a state of daydreaming) or due to an intensely engaged but variable (across individuals) processing of a movie sequence. For example, an art film may demand an intense intellectual effort from viewers. Nonetheless, ISC may be low because we might expect individual viewers to respond very differently to the same highly engaging material.

It is expected that some of the mental faculties engaged with the processing of a film will differ across film genres (e.g., drama, thriller, comedy). A highly emotional film is likely to engage the emotional systems of the brain, for example, while a highly contemplative movie would probably engage regions of the prefrontal cortex. Even within the same movie the processing of different scenes may rely on the operations of different brain regions. This calls for a more refined application of the ISC analysis. Specifically, ISC can be used to assess the effectiveness of a movie sequence, separately, for each of several brain regions. Figure 8B, for example, plots ISC separately for visual and auditory areas across different movies. This kind of analysis may provide the investigator (or filmmaker) with information about the effectiveness of different aspects of the film. As another example, the results described above (Figure 6) suggest that the activity in some visual areas is affected only by the content of individual shots, regardless of the editing, or temporal order. Thus, measuring the ISC within these regions is likely to provide us with an assessment of the power of the image to evoke similar responses across viewers, but will be unrevealing as to the effectiveness of a juxtaposition of shots.

Additional information about audience engagement can be obtained by computing the ISC separately for different scenes in the movie. Figure 9A, for example, plots the ISC for each two-minute segment of the Hitchcock TV episode. This provides a dynamic, time-varying measure of engagement during the movie. In this example, the ISC increases dramatically near the end of the movie, corresponding to the climactic scene. Such a time-varying measure 


\section{A. Overal engagment across all brain areas}

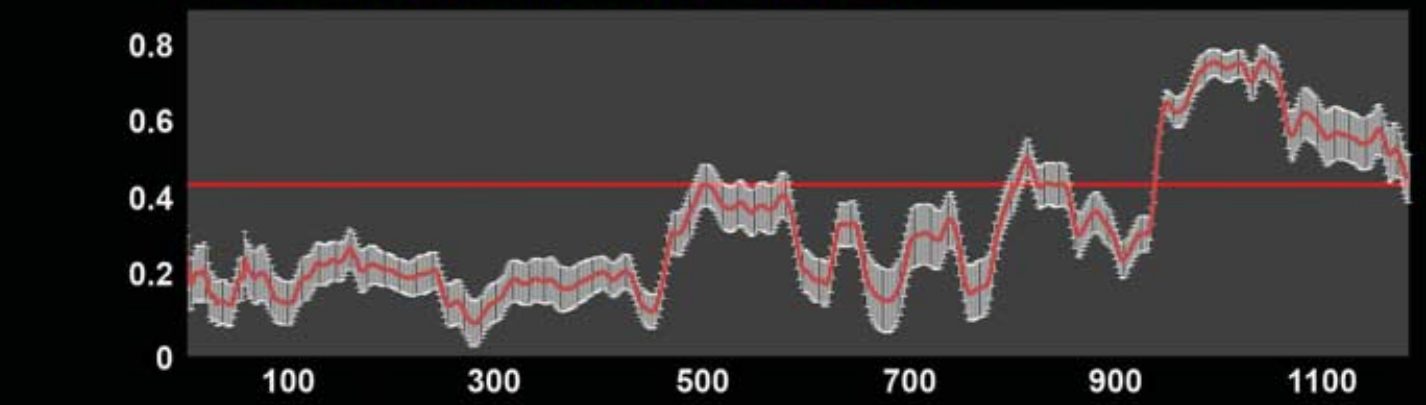

을

\section{B. Effectiveness of the movie sequence within the Auditory cortex}



\section{Effectiveness of the movie sequence within the DLPFC}

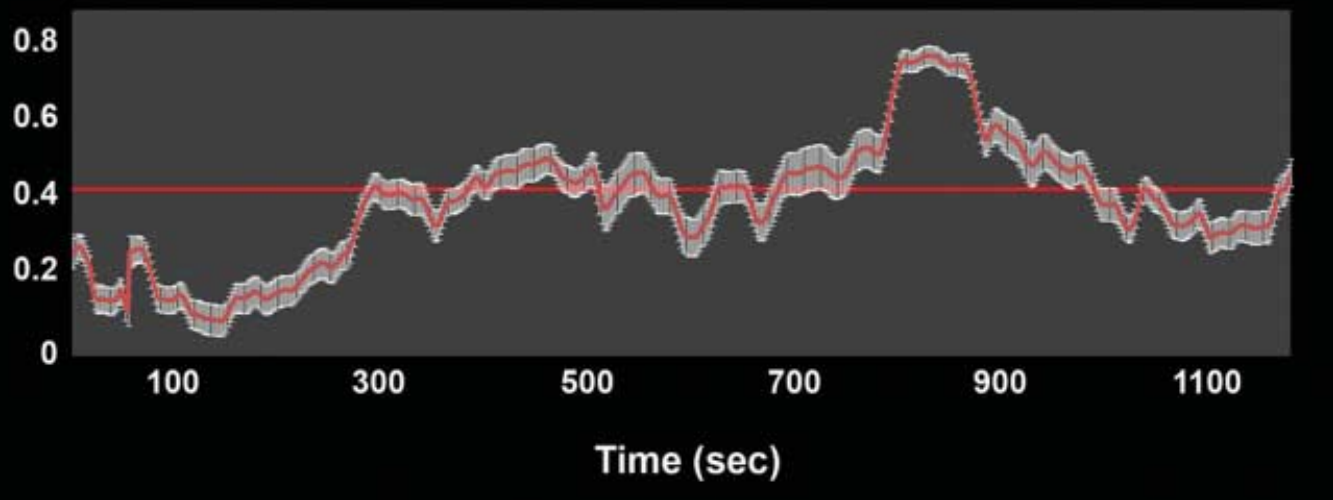

Figure 9. The evolution of the inter-subject correlation over time.

Figure 9A. The overall ISC (average across all cortex) for each two-minute segment of the Hitchcock movie. Red line marks the mean ISC, averaged over time.
Figure $9 B$. The ISC over time in auditory cortex during the Hitchcock movie.
Figure 9C. The ISC over time in the dorsolateral prefrontal cortex during the Hitchcock movie.

provides a new neuro-editing tool for assessing the moment-to-moment impact of a given film. Changes in engagement over time might be related to the director's intentions (as in this example) or unintentional. Measuring the evolution of ISC over time can provide filmmakers with information about the 
level of engagement in each scene or sequences of scenes. Detecting an unintentional reduction in the similarity of responses across viewers at a particular moment of the movie may suggest the need to further edit the scene to achieve the desired effect.

The temporal evolution of ISC can be refined further by measuring it separately for each of several brain regions. Figures $9 \mathrm{~B}$ and $9 \mathrm{C}$ plot the ISC over time in two brain regions, the early auditory cortex and dorsolateral prefrontal cortex (DLPFC, a region of the brain believed to be involved in certain higher cognitive functions) during the Hitchcock episode. The ISC in auditory cortex was high (average ISC value of 0.67 ) throughout the movie, attesting to the effectiveness of this particular soundtrack to induce similar responses across viewers throughout the entire duration of the film. On the contrary, the ISC in DLPFC was relatively low (average ISC value of 0.24 ), but increased substantially (>0.48) during a two-minute period (between 760 and $900 \mathrm{sec}$ in Figure 9C) about two-thirds of the way through the film. Additional research is needed to characterize the neuronal processing associated with such an increase of ISC in this area of the brain, which can contribute to a better understanding of the function of this brain region. This, in turn, might provide useful information to a filmmaker who is trying to achieve a certain impact on the audience.

Finally, so far we have focused only on the similarity in response time courses across all viewers while watching the same movie, ignoring individual and inter-group differences. The data presented in this article was obtained from college students, including approximately 50 percent men, 50 percent women, and 30 percent minorities. Thus, our results represent the average similarity across this heterogeneous group. Such similarity is expected given that all viewers are still part of a similar age group, are all experienced film viewers, and overall should perceive and interpret this particular set of films in a similar way. ${ }^{9}$ This finding is in agreement with an externalist philosophical stance that rejects the view that mental states represent an "intrinsic idiosyncratic property of mental life," and stresses the central role that the external environment plays in shaping our thoughts, intentions, and behaviors under different circumstances (Vygotsky 1962 [1934]; Wittgenstein 1951). However, in addition to these similarities, it is expected that the effects of a given film would vary across different individuals and target groups. Different spectators may perceive and interpret the same situation in various and sometimes opposite ways. Thus, the ISC analysis of brain activity can also serve as a measurement of systematic differences in how various groups of individuals (defined by age, gender, sexual preference, ethnicity, cultural background, etc.) respond to the same film. Measuring the ISC for different cultural groups may allow us to study the underlying neuronal substrates that correlate with inter-cultural differences. Moreover, it would allow us to assess the impact of a given film on different target groups. 


\section{Conclusion}

In this article we introduced a new paradigm-inter-subject correlation of brain activity-for measuring the effect of films on viewers' minds. This paradigm may pave the way to an innovative research approach we might call "neurocinematic" studies. Indeed, one of the goals of Projections: The Journal for Movies and Mind is to offer a platform for scholarly exchange between film studies and neuroscience (see, for example, Konigsberg 2007). The association between cinema and cognitive neuroscience is part of a larger endeavor that looks for connections between neuroscience and art (Kim and Blake 2007; Kawabata and Zeki 2004; Livingstone 2002; Ramachandran and Hirstein 1999; Zeki 1999). We propose that ISC may be of use to both film theorists and the film industry by providing a quantitative, neuroscientific assessment of viewers' engagement with a film. ISC is only one example of a growing trend in neuroscience to study the human brain under a more realistic and natural setting (Bartels and Zeki 2004a, 2004b; Haxby et al. 2001; Mobbs et al. 2006; Spiers and Maguire 2007a, 2007b; Wilson, Molnar-Szakacs, and lacoboni 2007; Zacks et al. 2001; Zeki 1998). These and other studies may provide the emerging field of neurocinematics with new tools for studying different aspects of films and filmmaking.

We should also note that a cognitivist approach to film is by no means a new theoretical path for film studies. In fact, it has been quite a dominant method of exploration since the 1980s. Film scholars, including Gregory Currie, Torben Grodal, Trevor Ponech, David Bordwell, Noël Carroll, and Murray Smith have written extensively on film perception, recognition, interpretation, and comprehension through the prism of understanding human mental processes. Bordwell and Carroll (1996) characterize the tool of cognitivism as a theoretical stance, which "seeks to understand human thought, emotion, and action by appeal to processes of mental representation, naturalistic processes, and (some sense of) rational agency" (Bordwell and Carroll, 1996: xvi). We propose that ISC analysis can contribute to the cognitive movement in film theory, analogous to contributions that neuroscience has made to cognitive and social psychology.

As with any innovation, one should proceed with caution. The ISC method provides us with a new way for assessing one essential aspect of films; that is, the level of control a given movie has on viewers' minds. It is clear to us that the similarity of responses is not the only measure, and not even the central measure, for assessing the quality of a movie sequence. Thus, the ISC measurement should probably not be used to evaluate the aesthetic, artistic, social, or political value of movies. As argued above, different filmmakers differ in the level of control they choose to impose on viewers, and our methods are not designed to judge this, but rather to measure the effect of a given film on different target groups. Thus, the critical evaluation of each film is outside 
the domain of this research, and should be left for the audience and the film critics. Moreover, the initial data presented here surely opens numerous questions, which call for new experiments. We anticipate that a more systematic exploration of the responses of different brain regions, from individuals in different target groups to various types of films, will shed new light on the burgeoning study of mind and cinema.

\section{Acknowledgments}

We thank Ira Konigsberg and Projections: The Journal for Movies and Mind for encouraging us to write this integrative article. We thank our neuroscientist colleagues Randolph Blake, David Carmel, Ifat Levy, Daniela Schiller, and our film studies colleagues and filmmakers Richard Allen, Ra'anan Alexandrowicz, Dan Chyutin, Zohar Lavi, and Gal Raz for their helpful suggestions and comments on the manuscript. Special thanks to Rafael Malach who supported this project from its inception. Funding for Uri Hasson was provided by the International Human Frontier Science Program Organization long-term fellowship.

Uri Hasson is a research scientist at the Center for Neural Science and Department of Psychology at New York University. B.A. Philosophy of mind (1994). M.A. Cognitive sciences (1999). Ph.D. Neurobiology, Weizmann Institute of Science (2004). Rothschild Fellowship (2004). Human Frontier Long-Term fellowship (2006).

David J. Heeger is a Professor of Psychology and Neural Science at New York University. B.A. Mathematics, University of Pennsylvania (1983). Ph.D. Computer Science, University of Pennsylvania (1987). Postdoctoral Research, MIT (1987-1990). Assistant Professor, Stanford University (1991-1998). Associate Professor, Stanford (1998-2002). David Marr Prize in computer vision (1987). Alfred P. Sloan Research Fellowship (1994). Troland Award, National Academy of Sciences (2002). Margaret and Herman Sokol Faculty Award in the Sciences, New York University (2006).

Ohad Landesman is a PhD student at the Department of Cinema Studies at New York University, from which he holds a Masters degree too. B.A in Film and Television and a LL.B (Bachelor of Laws) from Tel-Aviv University.

Nava Rubin is an associate Professor of Neural Science at New York University. B.A and M.A in physics, Hebrew University, Israel. PhD in neuroscience, Hebrew University, Israel. Post doc at Harvard University. Rothschild Fellowship and a Fulbright Junior Researcher Fellowship. McDonnell-Pew investigator-initiated award in Cognitive Neuroscience (1995). Alfred P. Sloan Fellowship (2000). 
Barbara Knappmeyer is research scientist at the Department of Psychology and Center for Neural Science at New York University. State Examination in Biology and Mathematics, University of Tübingen (1999). Doctoral Student, Max Planck Institute for Biological Cybernetics, Tübingen, Germany (1999-2003). Dr. rer. nat. with honors "summa cum laude,"University of Tübingen, Germany (2004).

Ignacio Vallines is a lecturer and research scientist at the department of experimental psychology of the University of Munich. Licenciado Superior in Psychology and Cognitive Science from the Universidad Complutense de Madrid (2001), and Ph.D. in Psychology, University of Regensburg (2007). 


\section{Notes}

${ }^{1}$ Montage editing, that originated as a concept in the Russian cinema of the 1920 , achieves meaning through the juxtaposition of spatially and/or temporally unrelated shots. This relationship often relies on conflict rather than individuating meaning from each shot.

${ }^{2}$ See, for example, the writings of Soviet theoretician and filmmaker Sergei Eisenstein, who embraced the dominant trend of Pavlovian behaviorism in the 1920 s to direct the thought processes and emotions of the spectator in a series of "shocks" and attractions. Eisenstein describes montage editing in his own Strike (1925) as "snatching fragments from our surroundings according to a conscious and predetermined plan calculated to launch them at the audience in the appropriate combination, to subjugate it to the appropriate association with the obvious final ideological motivation" (Eisenstein 1925: 57).

${ }^{3}$ One such effort to understand our narrative engagement in film by using cognitive tools was made by David Bordwell (1985). Bordwell analyzed how we make inferences and form hypotheses by embracing a constructivist theory of perception.

${ }^{4}$ For a systematic discussion on how directors direct our attention by aesthetically manipulating composition, movement and contrast of color and tonality, see Bordwell and Thompson 2008: 140-53. Similarly, Noël Carroll (1996: 84-86) argues that movies have more effective devices for directing the attention than theatre does, and lists three formal devices for achieving such purpose through camera positioning: indexing (camera moving toward an object), bracketing (indicating importance by the act of frame inclusion/exclusion), and scaling (the ability to change the scale of objects). In a cognitive analysis of attention guidance in film, Ira Konigsberg points to the manner in which techniques of focus (deep focus, shallow focus, follow focus, etc.) control our attention to an image in motion. "Part of the pleasure of viewing a film," Konigsberg explains, "is having our attention guided in an immediate and controlled manner, seeming to have the camera do the looking for us-following the objects of definition one after the other, we impose on them some kind of relationship and, ultimately, some kind of narrative" (2007: 13).

${ }^{5}$ The fact that the ISC results for the Curb Your Enthusiasm episode come closer to those for the unstructured segment of reality might be attributed, in part, to the fact that this TV series is often shot without a script.

${ }^{6}$ Much of the "Apparatus" film theory written in the 1970 (often termed as "Grand Theory") tried to provide an explanation for the spectator's fascination with Hollywood cinema by relying heavily on the assumption that its cinematic images generate an illusion of reality. Respectively, the prevailing view of spectatorship emphasized unconscious processes of identification and their seemingly inescapable effects on the viewer. See, for example, the writings of Jean-Louis Baudry (1974) about the illusion generated by the apparatus or the suture model of the shot-reverse shot mechanism proposed by Daniel Dayan (1974).

${ }^{7}$ In fact, the idea that a film cannot be a legitimate work of art if it is nothing more than a mechanical reproduction dominated the early thinking about cinema. Such, for example, were the efforts of Rudolf Arnheim (1957), who had to refute the prejudice against the medium by proving that the most important feature of film is to manipulate reality, to rearrange the profilmic event, and not to merely record it.

${ }^{8}$ One infamous historical example of a tight aesthetic control over the viewer is Leni Riefenstahl's Nazi propaganda films Triumph of the Will (1935) or Olympia (1938), in which groundbreaking nonfiction techniques are used in delivering a manipulative political message.

${ }^{9}$ A similar understanding of the responses of viewers to film involving an assumption of inter-subjectivity has been expressed by David Bordwell (1985). Bordwell suggested that all viewers make hypotheses while watching a film, based on affirmed knowledge that is 
taken as a "shared social resource." Ira Konigsberg, in a similar fashion, applies the neuroscience investigation of "mirror neurons" to study empathy in film with the supposition that "our brains function much the same when we view a movie, but with enough latitude to allow us some unique feelings and reactions" (Konigsberg 2007: 15).

\section{References}

Arnheim, Rudolf. 1957. Film as Art. Berkeley: University of California Press.

Bartels, Andreas, and Semir Zeki, 2004a. "The Chronoarchitecture of the Human Brain—Natural Viewing Conditions Reveal a Time-Based Anatomy of the Brain." Neuroimage 22 (1): 419-33.

- 2004b. "Functional Brain Mapping During Free Viewing of Natural Scenes. Human Brain Mapping 21 (2): 75-85.

Baudry, Jean-Louis. [1970] 1974. "Ideological Effects of the Basic Cinematographic Apparatus." Film Quarterly 28 (2): 39-47.

Bazin, André. 1967. What Is Cinema? Essays. Berkeley: University of California Press.

Bordwell, David. 1985. Narration in the Fiction Film. Madison: University of Wisconsin Press.

Bordwell, David, and Kristin Thompson. 2008. Film Art: An Introduction. New York: McGraw Hill.

Bordwell, David and Noël Carroll. 1996. Post-Theory Reconstructing Film Studies. Madison: University of Wisconsin Press.

Carroll, Noël. 1996. Theorizing the Moving Image. New York: Cambridge University Press.

Crick, Francis. 1994. "The Astonishing Hypothesis." The Journal of Consciousness Studies 1 (1): 10-16.

Damasio, Antonio. 2000. The Feeling of What Happens: Body and Emotion in the Making of Consciousness. New York: Harcourt.

Dayan, Daniel. 1974. "The Tutor-Code of Classical Cinema." Film Quarterly 28 (1): 22-31.

Douchet, Jean. 1985. "Hitchcock and His Audience." Pp. 150-157 in Cahiers du Cinéma, the 1950s: Neo-Realism, Hollywood, New Wave, ed. Jim Hillier. Cambridge: Harvard University Press.

Eisenstein, Sergei. 1925. "The Problem of the Materialist Approach to Form. Pp. 59-64 in The Eisenstein Reader, ed. Richard Taylor. London: British Film Institute.

Furman, Orit, Nimrod Dorfman, Uri Hasson, Lila Davachi, and Yudi Dudai. 2007. "They Saw a Movie: Long-Term Memory for an Extended Audiovisual Narrative." Learning and Memory 14 (6): 457-67.

Golland, Yuli, Shlomo Bentin, Hagar Gelbard, Yoav Benjamini, Ruth Heller, Yuval Nir, Uri Hasson, and Rafael Malach. 2007. "Extrinsic and Intrinsic Systems in the Posterior Cortex of the Human Brain Revealed During Natural Sensory Stimulation." Cereb Cortex 17 (4): 766-77.

Hasson, Uri, Eunice Yang, Ignacio Vallines, David J. Heeger, and Nava Rubin. 2008. A Hierarchy of Temporal Receptive Windows in Human Cortex. J Neuroscience 28 (10): 2539-50.

Hasson, Uri, Orit Furman, Dav Clark, Yudi Dudai, and Lila Davachi. 2008. "Enhanced Intersubject Correlations During Movie Viewing Correlate with Successful Episodic Encoding." Neuron 57 (3): 452-62.

Hasson, Uri, and Rafael Malach. 2005. "Human Brain Activation During Viewing of Dynamic Natural Scenes." Novartis Foundation Symposium NF 270: 203-212; discussion 212-16, $232-37$.

Hasson, Uri, Yuval Nir, Ifat Levy, Galit Fuhrmann, and Rafael Malach. 2004. "Intersubject Synchronization of Cortical Activity During Natural Vision." Science 303 (5664): 1634-40.

Haxby, James V., M. Ida Gobbini, Maura L. Furey, Alumit Ishai, Jennifer L. Schouten, and Pietro Pietrini. 2001. "Distributed and Overlapping Representations of Faces and Objects in ventral Temporal Cortex." Science 293 (5539): 2425-30. 
Heeger, David J., and David Ress. 2002. "What Does fMRI Tell Us About Neuronal Activity?" Nat Rev Neuroscience 3 (2): 142-51.

Huettel, Scott A., Allen W. Song, and Gregory McCarthy. 2004. Functional Magnetic Resonance Imaging. Trans. Allen W. Song and Gregory McCarthy. Sunderland, MA: Sinauer.

Kanwisher, Nancy, Josh McDermott, and Marvin M. Chun. 1997. "The Fusiform Face Area: A Module in Human Extrastriate Cortex Specialized For Face Perception.” J. Neuroscience 17 (11): 4302-11.

Kanwisher, Nancy, and Morris Moscovitch. 2000. "The Cognitive Neuroscience of Face Processing: An Introduction." Cognitive Neuropsychology 17 (1-3): 1-11.

Kawabata, Hideaki, and Semir Zeki. 2004. "Neural Correlates of Beauty." J Neurophysiology 91 (4): 1699-705.

Kim, Chai-Youn, and Randolph Blake. 2007. "Brain Activity Accompanying Perception of Implied Motion in Abstract Paintings." Spatial Vision 20 (6): 545-60.

Konigsberg, Ira. 2007. "Film Theory and the New Science." Projections: The Journal for Movies and Mind 1 (1): 1-24.

Ledoux, Joseph. 1998. The Emotional Brain: The Mysterious Underpinnings of Emotional Life. New York: Simon \& Schuster.

Livingstone, Margaret. 2002. Vision and Art : The Biology of Seeing. New York: Harry N. Abrams. Mobbs, Dean, Nikolaus Weiskopf, Hawkin C. Lau, Eric Featherstone, Ray J. Dolan, and Chris D. Frith. 2006. "The Kuleshov Effect: The Influence of Contextual Framing on Emotional Attributions." Soc Cogn Affect Neuroscience 1 (2): 95-106.

Ramachandran, V. S., and William Hirstein. 1999. "The Science of Art: A Neurological Theory of Aesthetic Experience." Journal of Consciousness Studies 6 (6-7): 15-51.

Spiers, Hugo J., and Eleanor A. Maguire. 2007a. "Decoding Human Brain Activity During Real-World Experiences." Trends in Cognitive Science 11 (8): 356-65.

Vygotsky, Lev S. [1934] 1962. Thought and Language. Trans. Eugenia Hanfmann and Gertrude Vakar. Cambridge: MIT Press.

Wilson, Stephen M., Istvan Molnar-Szakacs, and Marco lacoboni. 2008. "Beyond Superior Temporal Cortex: Intersubject Correlations in Narrative Speech Comprehension." Cerebral Cortex 18 (1): 230-42.

Wittgenstein, Ludwig. 1951. Philosophical Investigations. Oxford, Blackwell Publishing.

Zacks, Jeffrey M., Tod S. Braver, Margaret A. Sheridan, David I. Donaldson, Abraham Z. Snyder, John M. Ollinger, Randy L. Buckner, and Marcus E. Raichle. 2001. "Human Brain Activity Time-Locked to Perceptual Event Boundaries." Nature Neuroscience 4 (6): 651-55.

Zeki, Semir. 1998. "Art and the Brain." Daedalus: Proceedings of the American Academy of Arts and Sciences 127 (2): 71-104.

Zeki, Semir. 1999. Inner Vision: An Exploration of Art and the Brain. New York: Oxford University Press.

\section{Filmography}

Chaplin, Charlie. 1917. The Adventurer. USA.

- 1931. City Lights. USA.

David, Larry. 2000. Curb Your Enthusiasm (TV series). USA.

Eisenstein, Sergei, M. 1925. Strike (Stachka). Soviet Union.

Hitchcock, Alfred. 1961. Bang, You're Dead! (from Alfred Hitchcock Presents TV series). USA. Leoni, Sergio. 1966. The Good, the Bad and the Ugly (Buono, il brutto, il cattivo). Spain and Italy. Riefenstahl, Leni. 1935. Triumph of the Will (Triumph des Willens). Germany.

_. 1938. Olympia Part Two: Festival of Beauty (Olympia 2. Teil - Fest der Schönheit). Germany. 